MIDDLE EAST STUDIES ASSOCIATION BULLETIN

Vol. 8, No. 2, May 31, 1974

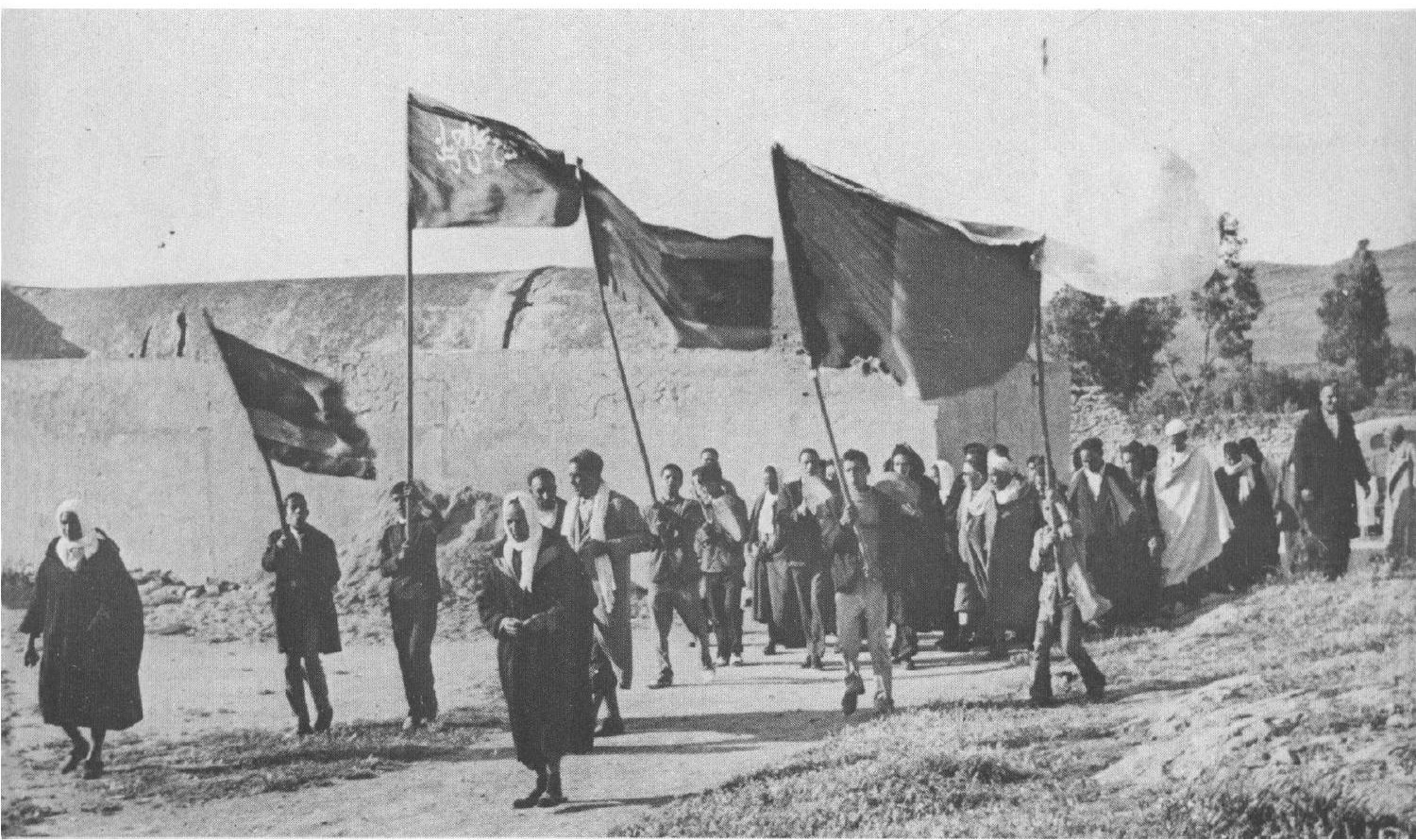

IMPORTANT ANNOUNCEMENT

PRELIMINARY PROGRAM FOR THE MIDDLE EAST STUDIES

ASSOCIATION EIGHTH ANNUAL MEETING................ 34

NOMINATIONS FOR THE 1974 ELECTION.............. 84

DUES FOR $1975 \ldots \ldots \ldots \ldots \ldots \ldots \ldots \ldots \ldots \ldots \ldots \ldots \ldots$ 


\section{COPYRIGHT 1974}

by Middle East Studies Association of North America, Inc. Headquarters and Secretariat, New York University Hagop Kevorkian Center for Near Eastern Studies 50 Washington Square South, New York, New York 10003

Views expressed in the Middle East Studies Association Bulletin are those of the authors and do not necessarily represent the Association.

US ISSN $0026-3184$ 


\section{MIDDLE EAST STUDIES ASSOCIATION BULLETIN}

Vol. 7, No. 3, May 31, 1974

This issue's cover shows a group of men from ${ }^{\text {Testour bearing }}$ the banners of Testour's patron saint, Sidi Ali el Arian, on a collective visit to the shrine of Sidi Ali Shaib near Fahs, spring 1973. 


\section{MIDDLE EAST STUDIES ASSOCIATION}

HONORARY FELLOWS

Philip K. Hitti

Aziz Atiya
S.D. Goitein

Sir Hamilton Gibb (d. 1971)

\section{OFFICERS}

\section{President}

G.E. von Grunebaum (Hon.) - 1967

(d. 1972)

Morroe Berger - 1967

George F. Hourani - 1968

R. Bayly Winder - 1969

William M. Brinner - 1970

John S. Badeau - 1971

Malcolm Kerr - 1972

Charles Issawi - 1973

Leonard Binder - 1974

\section{Directors}

William R. Polk - 1967

William D. Schorger - 1967

Charles Adams - 1967-68

William M. Brinner - 1967-68

Richard $H$. Nolte - 1967-69

R. Bayly Winder - 1967-69

Roderic Davison - 1968-70

G.E. von Grunebaum - 1968-70

Thomas Naff - 1969

Roger M. Savory - 1969-71

Farhat J. Ziadeh - 1969-71

\section{Vice President}

John S. Badeau - 1967

Charles Issawi - 1968

Ma 1co1m Kerr - 1969

Dankwart A. Rustow - 1970

Charles J. Adams - 1971

L. Carl Brown - 1972

Roger M. Savory - 1973

Richard N. Frye - 1974

\section{Executive Secretary and Treasurer}

I. William Zartman - 1967-68-69-70-71-72-73

Dankwart A. Rustow (Acting) - Spring 1969

Nicholas S. Hopkins (Acting) - 1973-74

Assistant to the Executive Secretary

Barbara Moutsatos - 1967-70

Georgi Hopkins - 1970-71

Christine Eickelman - 1971-72

Elana Mashberg - 1973-

INTERNATIONAL JOURNAL OF MIDDLE EAST STUDIES

Editor

Stanford J. Shaw, University of California, Los Angeles

\section{Editorial Board}

William M. Brinner, University of California, Berkeley

Lloyd Fallers, University of Chicago

oleg Grabar, Harvard University

Albert Hourani, Saint Anthony's College, Oxford

Charles Issawi, Columbia University

Nikki R. Keddie, University of California, Los Angeles

Malcolm Kerr, University of California, Los Angeles

Dankwart Rustow, City University of New York

R.B. Serjeant, Cambridge University

I. William Zartman, New York University 


\section{TABLE OF CONTENTS}

ARABIC STUDIES IN SPAIN TODAY

by Mike1 de Epa1za........................ 1

THE MIDDLE EAST IN AMERICAN HIGH SCHOOL CURRICULA:

A KANSAS CASE STUDY

by Michael $w$. Suleiman..................... 8

A BIBLIOGRAPHY OF MIDDLE EASTERN STATISTICAL DOCUMENTS (1945)

by Virginia $\mathrm{H}$. Gibbons..................... 20

PRELIMINARY PROGRAM FOR MESA EIGHTH ANNUAL MEETING . . . . . 34

MINUTES OF THE BOARD OF DIRECTORS MEETING SUMMARY. . . . . 44

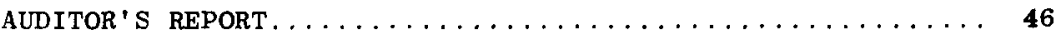

THE BRITISH SOCIETY FOR MIDDLE EASTERN STUDIES . . . . . . 48

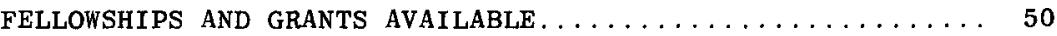

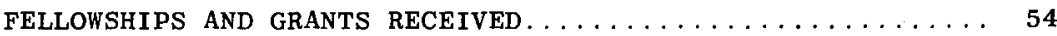

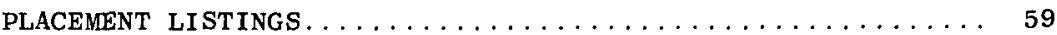

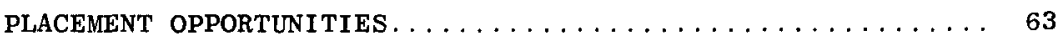

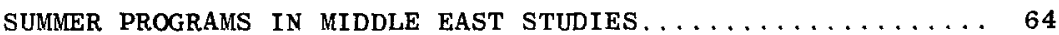

INTERNATIONAL VISITORS $\ldots \ldots \ldots \ldots \ldots \ldots \ldots$

THE MIDDLE EAST IN OTHER ASSOCIATIONS' MEETINGS . . . . . . 68

CONFERENCES . ........................... 70

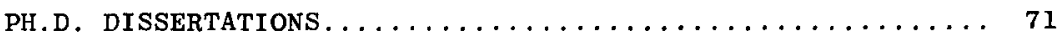

NEWS OF THE MEMBERS $\ldots \ldots \ldots \ldots \ldots \ldots \ldots \ldots \ldots \ldots \ldots \ldots \ldots$

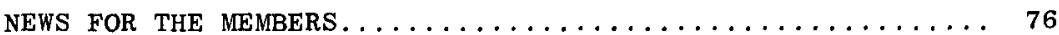

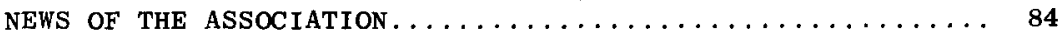

IMPORTANT ANNOUNCEMENTS

PRELIMINARY PROGRAM FOR MIDDLE EAST STUDIES

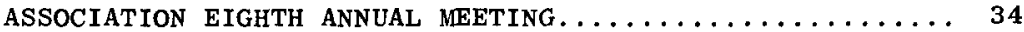

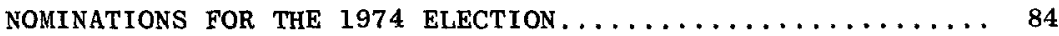

DUES FOR $1975 \ldots \ldots \ldots \ldots \ldots \ldots \ldots \ldots \ldots \ldots \ldots \ldots \ldots \ldots$ 